\title{
Sea spray aerosol and wave energy dissipation in the surf zone
}

\author{
M.J. Francius ${ }^{1}$, J. Piazzola ${ }^{2}$, P. Forget ${ }^{2}$, O. Le Calve ${ }^{2}$ and J. Kusmierczyk-Michulec ${ }^{1}$ \\ ${ }^{1}$ TNO Defense, Safety and Security \\ P.O. Box 96864, 2509 JG The Hague, The Netherlands \\ ${ }^{2}$ LSEET- Southern University of Toulon-Var \\ BP 56, 83162 La Valette du Var-Cedex, France
}

\begin{abstract}
Results from a quantitative model for the prediction of the sea-salt mass flux produced in the surf zone are presented in this paper. The model relates the surf zone sea salt mass flux to the amount of wave energy dissipated in the surf zone. In order to apply this aerosol emission model, a wave numerical model is required to obtain estimates for the total wave energy dissipated in the surf zone, as well as for the width of the surf zone. In the present work, we show using different wave models that the aerosol emission model is not sensitive to the details of the formulation of the wave model, provided a clear definition for the width of surf zone is adopted and the calibration of the numerical models is properly done.
\end{abstract}

Keywords: surf zone, sea spray aerosol, wave energy dissipation, sea-salt mass flux

\section{INTRODUCTION}

Over the past 10 years, there has been an increasing interest in the assessment of the effect of the seasalt aerosols (SSA) produced in the surf zone on the downwind SSA concentrations, because these sea spray droplets play a very important role in a variety of atmospheric processes, including visibility, transport of pollutants and bacteria, and electro-optical propagation in coastal areas. Although the determination of the surf zone SSA production flux is not well quantified due to lack of any significant data base on SSA surf production events, recent studies indicate that the local wave field and the local bottom bathymetry would be as important as the characteristics of the local wind system to determine the rate of SSA production across the surf zone $e^{1,2}$.

Prior to the studies ${ }^{3,4}$ addressing the determination of the surf source function or size-distribution of SSA production flux, measurements of near-surface sea-salt mass fluxes at several stations along the southern Baltic coasts have been used to develop a simplified parameterization for the total sea-salt mass emission flux, $F_{e}$, due to the SSA production in the surf zone, with the total amount of wave energy dissipated, $d E$, due to depth-induced wave breaking across the surf zone ${ }^{5,6,7}$. From scaling arguments on the decrease of potential wave energy due to wave breaking, the amount of air entrained into water and the characteristics of the resulting surface bubbles density after their subsequent rising to the sea surface, the relation was obtained as follows,

$$
F_{e}=A \cdot d E^{3 / 4}+B
$$

where $F_{e}$ is in units of $\mu \mathrm{g} \cdot \mathrm{m}^{-2} \cdot \mathrm{s}^{-1}, A$ and $B$ are constants to be determined experimentally and the total amount of wave energy dissipated, $d E$ in units of $\mathrm{kg} . \mathrm{s}^{-3}$, is defined as the spatially averaged value across the width of the surf zone of the local rate of wave energy dissipation due to depth-induced wave

Atmospheric Optics: Models, Measurements, and Target-in-the-Loop Propagation,

edited by Stephen M. Hammel, Alexander M. J. van Eijk, Michael T. Valley, Mikhail A. Vorontsov,

Proc. of SPIE Vol. 6708, 67080N, (2007) $\cdot 0277-786$ X/07/\$18 $\cdot$ doi: 10.1117/12.734261

Proc. of SPIE Vol. $670867080 \mathrm{~N}-1$ 
breaking. With this approach the local values of the wave energy dissipation were computed using a numerical wave energy propagation (WEP) model describing the decrease of the total wave energy across the surf zone due to depth-induced wave breaking and bottom friction effects on the incoming random waves. Using estimates of $F_{e}$ from two experiments on the Baltic coasts, Petelski and Chomka obtained slightly different values for one set of experimental measurements versus the other. For A and B, they found, respectively, 99.4 and 3.5 for the BAEX experiments and the values 90.5 and 12.6 for the TABEX experiments.

Recently, this approach has been further validated with comparisons between modeled data and experimental data obtained during three surf experiments undertaken as part of the Electro-Optical Propagation Assessment in Coastal Environments (EOPACE) efforts ${ }^{8}$. In this study the general conclusions drawn by Petelski and Chomka ${ }^{6,7}$ about the dependency on the total sea-salt mass emission flux with incoming wave parameters and bottom bathymetry have been more or less confirmed. However, large differences in the predicted local and total values of sea-salt mass emission fluxes have been reported depending on the choice of WEP model to calculate the local rates of wave energy dissipation across the surf zone. In the case they present (see their figure 3), two WEP models have been used and it has been claimed that one model was superior to the other on the basis only of visual inspections of the predictions for the width of the surf zone, $L$, and magnitudes of the cross-shore profile of the sea-salt mass emission flux given by equation (1). We note that no details about the WEP models were presented and, further, that these results are somewhat in contradiction with past studies concerned with the hydrodynamics induced by random breaking waves on beaches.

In the present work we try to clarify which WEP model would yield the best predictions for the characteristics of the surf zone such as the width of the surf zone and the total amount of wave energy dissipated due to wave breaking. This paper presents the preliminary analysis of the data collected at Scripps pier in November 2006, during an experiment focusing primary at the quantitative determination of the SSA production flux due to breaking waves in the surf zone. The comparison for the SSA production flux between model predictions and the results of the experiments will be reported when the latter results will be available.

\section{WAVE ENERGY DISSIPATION IN THE SURF ZONE}

\subsection{Background information}

Recently, assessments of the surf source function based on measurements of the increase (compared to background) in size-distribution of SSA concentrations measured at the shore during onshore wind conditions have been reported ${ }^{3,}{ }^{4}$. The conversion from near-surface measurements of SSA concentrations to SSA flux at the sea surface (source function) requires that the width of the surf zone $L$ should be prescribed among other factors characterizing the surf-generated SSA plumes. Alternatively, the approach of Petelski and Chomka enables to predict the total sea-salt mass flux associated with the SSA production across the surf zone. This task can be achieved when the coastal bathymetry and the near shore incoming wave parameters are known and used as inputs to a WEP model, which provides predictions for the width of the surf zone, $L$, and the averaged amount of wave energy dissipated in the surf zone, $d E$. When these quantities are known, estimates of the surf-produced emission flux $F_{e}$ can be obtained using equation (1). Although estimates of the width of the surf zone $L$ can be obtained either by analyzing video recordings of the surf zone or by using a WEP model, it is a far more challenging problem to estimate the quantity $d E$ due to the complexity of the wave hydrodynamics in the surf zone.

Despite the complexity of the wave breaking process in the surf zone, realistic random wave transformation models have been successfully developed for the prediction of cross-shore variations in wave heights across the surf zone. In this paper we consider only parametric models which address explicitly the calculation of the wave energy dissipation across the surf zone. The parametric models aim at describing the effects of dissipative mechanisms on the cross-shore variation of the total wave energy or total variance of sea surface elevation. 


\subsection{Description of models used in this study}

Parametric models are generally based on the use of an energy conservation equation for random waves approaching a shore, in order to determine the cross-shore variation of the total wave energy per unit surface area across the surf zone. Assuming linear water wave theory to be valid and considering obliquely incident waves on a coastline with straight and parallel contours, the mean wave energy balance equation can be written in general form as,

$$
\frac{d}{d x}\left(E C_{g} \cos \theta\right)=-\varepsilon_{b}-\varepsilon_{f}
$$

where $x$ is the cross-shore coordinate, $E$ is the total wave energy per unit surface area, $C_{g}$ is the wave group velocity and $\theta$ is the mean angle of incidence of the wave field. The terms on the right hand-side of equation (2), $\varepsilon_{b}$ and $\varepsilon_{f}$, represent the contributions to the averaged (over time) value of the local dissipation of wave energy due to depth-induced wave breaking effects and bottom friction effects, respectively. Generally, the wave dissipation term due to bottom friction effects can be neglected compared with the dominant wave breaking dissipation, except in the shallowest water or run-up region of mildly sloping beach. In this study we are not concerned by the run-up region so that the function $\varepsilon_{f}$ will be neglected.

The local wave energy density and group velocity are usually given by linear water wave theory relationships, which have been proved to be remarkably accurate for narrow-banded waves with characteristic incident (in deep water) peak frequency $f_{p}=\omega_{p} / 2 \pi$. These relations can be written for the general case of finite depth as,

$$
\begin{aligned}
& E=1 / 8 \rho_{w} g H_{r m s}^{2} \\
& C_{g}=\frac{\omega_{p}}{2 k}\left(1+\frac{2 k h}{\sinh (2 k h)}\right)
\end{aligned}
$$

where $H_{r m s}$ is the root-mean-square (rms) wave height, $k$ is the local wave number associated with the peak frequency $f_{p}$ and $h$ is the local water depth (including the wave setup/setdown induced by wave breaking). In the above formulas, $\rho_{w}$ is the water density and $g$ is the acceleration of gravity. Note that in deriving the above relations, it is usually assumed that reflection of wave energy can be neglected and that the cross shore variations of the bottom profile occur on larger scales than that of the wave field.

In order to use equations (2), (3) and (4), a model for the dissipation function, $\varepsilon_{b}$, is required to close the formulation. In this paper we consider only closure models based on the analogy with periodic propagating bores following Battjes \& Janssen ${ }^{9}$ since the performance of more complicated advanced closure models is not essentially better than that of the models presented here. In Battjes and Janssen's model, the averaged rate of wave energy dissipation for a periodic wave bore is given by

$$
D_{\text {bore }}=\frac{f}{4} \rho_{w} g \frac{B^{3}}{d} H^{3}
$$

where $d$ is the mean depth, $f$ is the wave frequency, $\mathrm{H}$ is the wave-height and $B$ is a constant parameter to be determined from experimental data. In essence, the parameter $B$ represents the fraction of foam on the face of the bore and accounts for the differences in the various breaker types. 
For application to random waves with (offshore) peak frequency, $f_{p}$, propagating in the surf zone, the averaged rate of wave energy dissipation at water depth $h$ is given by

$$
\varepsilon_{b}=\frac{f_{p}}{4} \rho_{w} g \frac{B^{3}}{h}\left(\int_{0}^{\infty} H^{3} p_{b}(H) d H\right)
$$

where $p_{b}(H)$ is a function describing the distribution of breaking wave heights. Because there is no theory for describing this distribution function, each closure model requires further approximations to parameterize the distributions of wave heights both inside and outside the surf zone. In a random wave field, the distribution functions $p_{b}(H)$ and $p(H)$ describes statistically how many waves in the ensemble are breaking and non breaking, respectively. These functions are related to the total wave energy density, $E$, and the fraction of breaking waves, $Q_{b}$, by the following relations,

$$
\begin{aligned}
& E=\frac{1}{8} \rho_{w} g \int_{0}^{\infty} H^{2} p(H) d H=\frac{1}{8} \rho_{w} g H_{r m s}^{2} \\
& Q_{b}=\int_{0}^{\infty} p_{b}(H) d H
\end{aligned}
$$

In this paper, we have considered three different closure models that are described explicitly below. Two of these models, referred as $A$ and $B$, are based on the work of Battjes and Janssen'. The third one, model $C$, is the model proposed by Thornton and Guza ${ }^{10}$. Without entering into details, the three closure models are presented hereafter.

Model A: This model corresponds to the original approach of Battjes and Janssen ${ }^{9}$. Namely, the authors have assumed that the wave-height distribution function, $p(H)$, for both breaking and non breaking waves could be approximated with a Rayleigh distribution truncated at a maximum limiting height $H_{m}$, such that the heights of all waves which are broken or breaking at a given water depth $h$ are equal to $H_{m}$. This was shown to imply the following relation between $Q_{b}$ and the ratio $H_{r m s} / H_{m}$,

$$
\frac{1-Q_{b}}{\ln Q_{b}}=-\left(\frac{H_{r m s}}{H_{m}}\right)^{2}
$$

for $H_{r m s}<H_{m}$. Because $Q_{b}$ is implicitly given in equation (9) some iterations is needed to evaluate $Q_{b}$. Using equation (9) and the corresponding distribution of breaking wave heights yields the following relation for the mean rate of wave energy dissipation,

$$
\varepsilon_{b}=\frac{B^{3}}{4} \rho_{w} g f_{p} \frac{H_{m}^{3}}{h} Q_{b} \approx \frac{\alpha}{4} \rho_{w} g f_{p} H_{m}^{2} Q_{b}
$$

where $\alpha=B^{3}$. Note that the last approximation can be justified in shallow water where the relation $H_{m} / h=O(1)$ holds very often. In this model, the limiting height $H_{m}$ is given by the following expression with the peak wave number, $k_{p}=\omega_{p}{ }^{2} / g$, as

$$
H_{m}=\frac{0.88}{k_{p}} \tanh \left(\frac{\gamma}{0.88} k_{p} h\right)
$$


where $\gamma$ is an empirical parameter. The determination of this parameter is critical because it determines the fraction of breaking waves and the place where the breaking occurs via the maximum wave height $H_{m}$. The parameter $\alpha$ controls the level of the rate of wave energy dissipation. Setting $\alpha$ equal to 1 , an extensive calibration study ${ }^{11}$ for $\gamma$ based on both field and laboratory data reveals that this parameter depends weakly on the incident (offshore) wave steepness $s_{0}$ and independent of the beach slope (i.e., for planar beach) as

$$
\gamma=0.5+0.4 \tanh \left(33 s_{0}\right)
$$

This relation has been used in our study with the offshore wave steepness defined by $s_{0}=H_{r m s, 0} / \lambda_{p}$, where $H_{r m s, 0}$ is the offshore rms wave height and $\lambda_{p}=2 \pi / k_{p}$ is the wavelength associated with the peak frequency $f_{p}$.

In general, model $A$ has been proved to be successful at describing $H_{r m s}$ transformation on planar beaches (with middle slopes) in both field and laboratory conditions. However, this model has few disadvantages. One of them is that all wave breaking occur at the same wave height $H_{m}$, so that close to the shoreline the rate of wave energy dissipation is underestimated. The situation does not accurately describe the wave transformation close to the shoreline and consequently $H_{r m s}>H_{m}$, which is impossible from a physical point of view. For this reason, the model solution is forced such that $H_{r m s}=H_{m}$, i.e. a saturated inner surf zone is assumed.

Model $B$ : This model is a reformulation of model $A$, which was proposed by Baldock et al. ${ }^{12}$ in order to improve the predictions for the cross-shore variations in both the rms wave height and the fraction of broken waves on steep beaches or non-planar beaches characterized by unsaturated surf zone conditions. The principal difference with model $A$ is that Baldock et al. ${ }^{12}$ used a normal Rayleigh distribution for wave heights both outside and inside the surf zone rather than a truncated Rayleigh distribution. This approach allows explicit expressions for both the fraction of breaking waves and the rate of wave energy dissipation, which are given by

$$
\begin{aligned}
& Q_{b}=\exp \left[-\left(\frac{H_{m}}{H_{r m s}}\right)^{2}\right] \\
& \varepsilon_{b}=\frac{\alpha}{4} \rho_{w} g f_{p}\left(H_{m}^{2}+H_{r m s}^{2}\right) \exp \left[-\left(\frac{H_{m}}{H_{r m s}}\right)^{2}\right]
\end{aligned}
$$

Following Baldock et al. ${ }^{12}$, the standard expression (11) has been used in our study with $\gamma$ given by a slightly different relation ${ }^{13}$ as

$$
\gamma=0.39+0.56 \tanh \left(33 s_{0}\right)
$$

Also the value of $\alpha$ is set equal to 1 in equation (14), since this was found to apply over a wide range of beach slopes. Only on steep slopes, Baldock et al. ${ }^{12}$ found that the performance of their model was significantly improved as compared to that of model $A$.

Model C: In view of the discussion presented in section 1, we present here one of the models developed by Thornton and Guza ${ }^{10}$. This model has been considered by Chomka and Petelski ${ }^{6,7}$ in order to estimate the coefficients in equation (1). Thornton and Guza ${ }^{10}$ adopted a similar approach for the dissipation function but modeled the distribution of breaking wave height using empirical functions based on field data analysis. For the model considered here, the distribution function of breaking waves 
that is required to be used in equations (6) and (8) is expressed as a weighted Rayleigh distribution for all wave heights as:

$$
p_{b}(H)=W(H) p(H)
$$

where $p(H)$ is the classical Rayleigh distribution. The weighting function $W(H)$ is given by

$$
W(H)=\left(\frac{H_{r m s}}{\gamma h}\right)^{4}\left[1-\exp \left[-\left(\frac{H}{\gamma h}\right)^{2}\right]\right]
$$

with the empirical parameter, $\gamma=0.42$, determined from measurements in the surf zone on near planar beaches of the Californian coasts. We note in passing that the absence of dependence in the parameter $\gamma$ with the offshore wave steepness or the beach slope reflects the fact that this parameter has a different meaning than that of the previous models. Indeed, this parameter is related with the observed values of the ratio $H_{r m s} / h$ in the inner surf zone where it is constant under saturated surf zone conditions ${ }^{10,14}$. The above weighting function yields, in turn, an analytical expression for the dissipation function as

$$
\varepsilon_{b}=\frac{3}{16} \sqrt{\pi} \rho_{w} g f_{p} \frac{\alpha}{\gamma^{2} h^{3}} H_{r m s}^{5}\left[1-\left(1+\left(\frac{H_{r m s}}{\gamma h}\right)^{2}\right)^{-5 / 2}\right]
$$

In this model, $\alpha=B^{3}$ is assumed constant and is the only free parameter to be determined by comparison between predictions and observations, both outside and inside the surf zone. On the other hand, it is apparent from (18) that the parameters $\alpha$ and $\gamma$ are not independent since the ratio $\alpha / \gamma^{2}$ occurs as an isolated term. Thornton and Guza ${ }^{10,14}$ performed an extensive calibration study with both laboratory and field data. They obtain the optimal values for $B=\alpha^{1 / 3}$ equal to 0.8 and 1.5 for laboratory and field results, respectively.

For all the models presented above, the local depth $h$ is the sum of the bottom depth below still water level, $h_{0}$, and a variation in mean water level of the mean surface, $\eta$, that represents wave-driven effects which are associated with shoaling and breaking waves. This variation is usually called wave setup (setdown) when it is positive (negative). In our study, it has been calculated using the mean momentum balance across the surf zone in the form ${ }^{15}$

$$
\frac{\partial S_{x x}}{\partial x}+\rho g\left(h_{0}+\eta\right) \frac{\partial \eta}{\partial x}=0
$$

where the radiation stress component, $S_{x x}$, is calculated according to linear wave theory, namely with

$$
S_{x x}=E \cdot\left[\left(2 n-\frac{1}{2}\right) \cos ^{2} \theta+\left(n-\frac{1}{2}\right) \sin ^{2} \theta\right]
$$

where $n=C_{g} / C$ is the ratio of the local group velocity (see equation 4 ) to the local phase velocity $C=$ $\omega_{p} / k$. The local mean wave direction $\theta$ is found using Snell's law

$$
\sin \theta=\frac{C}{C_{0}} \sin \theta_{0}
$$


where the subscripts 0 refers to a further offshore position, and the local wave number $k$ associated with the peak frequency $f_{p}=\omega_{p} / 2 \pi$ is determined using the following dispersion relation

$$
\omega_{p}^{2}=g k \tanh (k h)
$$

With a known bathymetry profile $h_{0}(x)$, incident (offshore) values for $f_{p}, H_{r m s, 0}$ and $\theta_{0}$, and estimates of the model parameters $\alpha$ and $\gamma$, the governing equations (2) and (19) can be numerically integrated from offshore to the shoreline to obtain the cross-shore variation of $H_{r m s}$ and $\eta$, as well as the corresponding variations of the wave energy dissipation $\varepsilon_{b}$ and the fraction of breaking waves $Q_{b}$. In this process, the frequency $f_{p}$ is supposed to be constant. In our application of the WEP models, a simple forward stepping numerical scheme has been used and it was found to be sufficiently accurate. Calculations proceed from the seaward end of the numerical grid to the shoreline. Due to the nonlinear nature of (2) and (19), the program iterates on the solutions until the updated values for the wave-induced changes in mean water level become very close to those found in the previous iteration. In our study, it was found that a few iterations are usually necessary for the difference in these estimates to become less than a millimeter. In the end, the position of the breaker line or equivalently the width of the surf zone, $L$, is determined to be at the location where $\eta$ starts to increase up to a maximum value at the shoreline. At this position, the sign of the slope of changes its sign and the gradient of the radiation stresses, $\partial S_{x x} \partial x$, vanishes. Thus, the position of the breaker line is found at the first maxima in the radiation stresses cross shore profile. This definition is consistent with many observations of wave setup/setdown both in laboratory and field studies ${ }^{15}$.

\section{WAVE DATA AND NUMERICAL RESULTS}

\subsection{Wave data}

Prior to the surf experiment that was carried out on November 2-29, 2006, in the near shore zone of the Scripps Institution of Oceanography (SIO) in La Jolla (California), beach profile surveys have been carried out on October, 29 and November, 42006 in the vicinity of Scripps's pier. Inspection of the surveyed bottom profiles reveals that near the pier bottom contours are relatively straight and parallel with a mean beach slope equal to 0.03 . In the numerical study presented in the next section, we have assumed that the cross-shore beach profiles measured prior to the experiment represent well the beach profiles that occurred during the surf experiment.

Wave data in the near shore zone of the Scripps' pier were collected during the surf experiment. The wave data consist of measurements obtained with a CDIP (Coastal Data Information Program) buoy operated by the Ocean Engineering Research Group at SIO. In addition we have used a high-resolution camera to photograph the propagation of the waves from outside the surf zone to the shoreline. .

The CDIP buoy 073 that is mounted at one pilling near the end of the Scripps pier is a pressure wave sensor, which was operating at a mean depth of approximately $6 \mathrm{~m}$. The sensor provides hourlyaveraged information about water depth and wave conditions just outside the surf zone. The wave data from this sensor are available trough internet (via, www.cdip.ucsd.edu). They consist of hourlyaveraged values for mean water depth, wave height, peak period and, also, frequency (energy) spectrum of the near shore wave field producing the surf zone at Scripps's pier. .

A video camera was installed on the deck of the pier and a CCD camera (Canon EOS 500) at the beach both looking over the surf zone in an approximately horizontal plane. Photographs of the surf zone were taken simultaneously with aerosol measurements as often as possible during the experiment. The use of these cameras was considered to investigate on the possibility to obtain estimates for the whitecap coverage in the surf zone from an image processing technique, which has previously been successfully applied to improve whitecap coverage estimation over the open ocean ${ }^{16,17}$. A geometrical transformation was developed to put the image pixels in perspective and regular graduations on the 
deck pier were used as geometric references to convert the pixel lengths into physical lengths. In addition the width of the surf zone could be fairly estimated on several occasions during the experiments.

\subsection{Calibration}

In practice, the results of the numerical model are very sensitive to the choice of the free parameters $\alpha$ and $\gamma$ (see section 2.2), which control the level of wave energy dissipation in a breaker and the fraction of breaking waves, respectively. In principle, they can be varied independently of each other. For model $A$ and model $B$, the parameter $\alpha$ was set equal to 1 and extensive calibration studies with both laboratory and field data yielded optimal values for the parameter $\gamma$ as given by (12) and (15). In contrast, for model $C$, the parameter $\gamma$ was determined from measured wave heights in the inner surf zone over a wide range of wave conditions at Torrey Pines (north of San Diego) beach, California ${ }^{10,14}$. Assuming $H_{r m s} / h$ is constant throughout the inner surf zone, they found the mean value of $\gamma$ was equal to 0.42 . Then, tuning of the model with comparison between modeled and measured wave heights across the surf zone yielded an optimal (mean) value for $B=\alpha^{1 / 3}$ equal to 1.5. Somewhat different values, $B$ ranging from 0.8 to 1.1 with $\gamma=0.43$, were reported by the same authors based on data at LeadBetter (Santa Barbara) beach, California.

Unfortunately, we could not deploy wave sensors across the surf zone because surf wave riders were always present inside the surf zone near the pier. Thus, we do not have wave heights measurements across the surf zone and, therefore, it is impossible to repeat here a calibration study based on the comparison between modeled and measured wave heights across the surf zone. So far, the only parameter characterizing the surf zone that has been measured is the width of the surf zone from video images analysis. Due to memory limitation of our camera we could not monitor the surf zone continuously during the whole period of the experiment. As a compromise, we have estimated a total number of 51 estimates (hourly averaged) for the width of the surf zone, $L$, from the analysis of all video images recorded during 8 days. Hence, we have adopted a two steps strategy in order to check, firstly, that the numerical implementation has been done properly for all models and, secondly, to analyze the sensitivity of the WEP models by varying one of the two free parameters of these models and testing the results against observed values for the width of the surf zone $L$.

To achieve the first task, we have considered the laboratory measurements of rms wave height, which were collected over a plane beach of constant slope equal to $1 / 40$ The data have been digitized from the original publication ${ }^{11}$. The incident wave conditions (case 10 in the table 1 of that paper) are reported here on the labeling of Figure 1. The results shown in Figure 1 have been obtained using the optimal values recommended for each model after extensive calibration studies. Namely, we took for models $A$ and $B, \alpha=1$ for both and $\gamma$ given by (12) and (15), respectively. For model $C$, we took $\gamma=0.42$ and $B=\alpha^{1 / 3}=0.8$, because we are dealing with a laboratory case. Figure 1 shows that there are almost no differences between model $A$ and $B$, while model $\mathrm{C}$ underestimate slightly the values of $H_{r m s}$ in comparison with the experimental data. Model $C$ underestimates the maximum value of the local rate of wave energy dissipation by a factor approximately equal to 2 in comparison with model $A$ and $B$. In this case, model $A$ seems to perform slightly better than model $B$ as far as the $H_{r m s}$ cross-shore profile is concerned. 

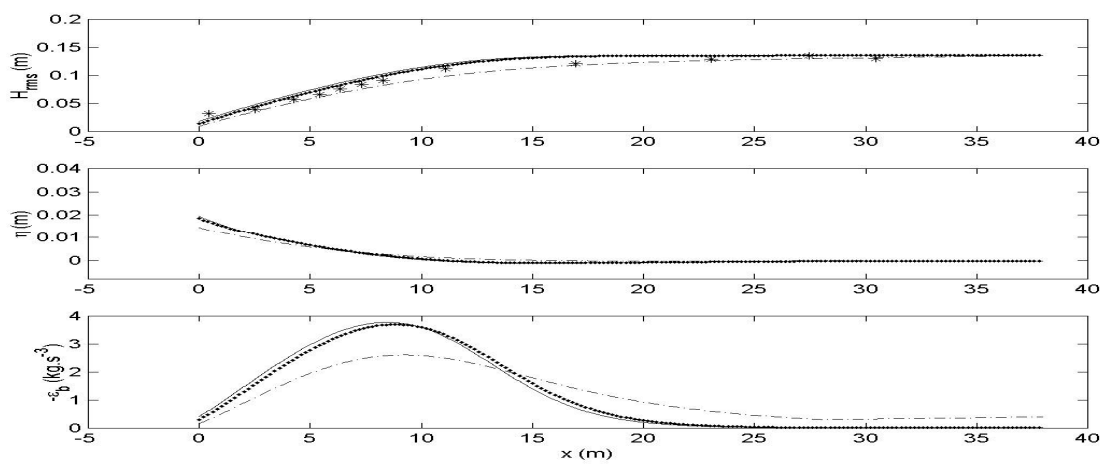

Figure 1. Experimental $H_{r m s}$ values (stars) and predicted profiles of $H_{r m s}$ (top), set-up (middle) and dissipation (bottom). Dotted line: model $A$; Solid line: model $B$; Dash-dotted line: model $C$. Offshore wave conditions: $H_{r m s}=0.136 \mathrm{~m}$ and $f_{p}=0.633 \mathrm{~Hz}$.

In a second step, the sensitivity of each model has been studied with our field data, using the measured bottom profile near Scripps's pier and the incident wave data of the buoy CDIP073 located outside the surf zone near the end of the pier. Actually, this model calibration study has been based on a comparison between measured and predicted values of the width of the surf zone. In this process, the numerical integration of the main equations has been repeated for different choices of $\alpha$ and $\gamma$ values, so that a maximum agreement is obtained between measured and modeled values. Note also that our definition of the width of the surf zone, $L$, based on the location of the maxima of the radiation stresses, $S_{x x}$, differs somewhat from that proposed by Chomka and Petelski ${ }^{6}$, which is based on a threshold value of 0.001 (without units in their paper) for the rate of wave energy dissipation $\varepsilon_{b}$. Applying their definition and assuming units of $\mathrm{kg} \mathrm{s}^{-3}$ would have yielded erroneous values for $L$ in the laboratory case presented before (see figure 1).

Applications of our WEP models to the selected cases (51 values) where measurements of $L$ are available have enabled us to find that the rms error between predicted $L$ values based on our definition and the observed values based on video images analysis goes through a minimum for model $A$ and $C$, except for model $B$, with the increase of the parameter $\alpha$ from 0.5 to 1.9 (taking $\gamma=0.42$ for model $C$, and $\gamma$ given by (12) and (15), respectively, for model $A$ and $B$. With model $B$, however, the rms error for $L$ increases monotonically for model $B$, although inspection of the predicted cross-shore profile reveals unrealistic values for the extreme values of the parameter $\alpha$. Thus, following Baldock et al. (1998), we have therefore used $\alpha=1$ and $\gamma$ given by (15) for model $B$, which yields a rms relative error equal to $36.2 \%$ for all 51 data points. In comparison, we have obtained the minimum rms errors values of $28.5 \%$ and $26 \%$, respectively, for model $A$ with $\alpha=1$ and $\gamma$ given by (12) and model $C$ for with $\alpha=1$ and $\gamma=0.42$.

Figure 2 shows the predictions of the three models considered here for the width of the surf zone as well as the experimental values determined from video images analysis. This shows that the general trend in the variation of $L$ is well predicted with the 3 models, except maybe for the data number ranging from 35 to 42 that correspond to a case when $L$ was constantly decreasing. The models tend to overestimate by more than a factor 2 the width of narrow surf zones (corresponding to the first 20 samples of our dataset). By removing these values from the dataset and repeating the above analysis by varying the parameter, we have obtained improved minimum rms error values, i.e $20 \%$ for model $\mathrm{A}$ with $\alpha=1.2,26 \%$ for model $B$ with $\alpha=1.0$ and $18 \%$ for model $\mathrm{C}$ with $\alpha^{1 / 3}=B=0.95$. 


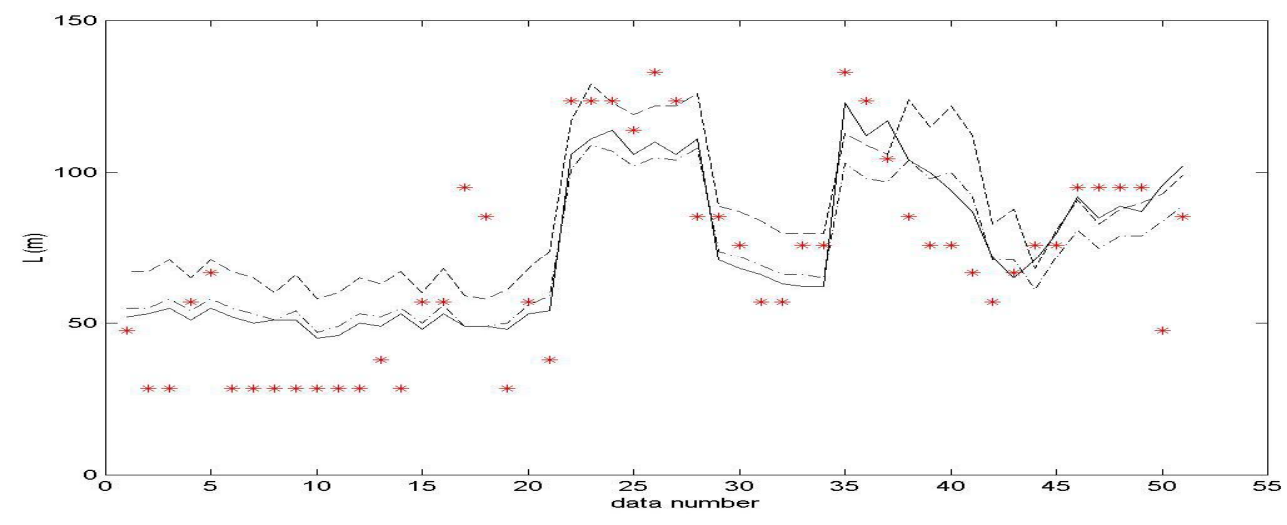

Figure 2: Comparison between predictions and observations of $\mathrm{L}$ values (51 experimental data) for three different WEP models. Dash-dotted line: model A with $\alpha=1.0$ and $\gamma$ given by (12); Dashed line: model B with $\alpha=1.0$ and $\gamma$ given by (15); Solid line: model $\mathrm{C}$ with $\alpha=1.0$ and $\gamma=0.42$; stars: experimental values.

The reasons why the models fail to predict small $\mathrm{L}$ values may be related to the fact that for those cases the surf zone was not saturated as assumed in models $A$ and $C$. Not all wave energy is dissipated and there maybe considerable wave energy reflection, when not enough breaking occur inside the narrow surf zone. Finally, we note that model $B$ was developed for unsaturated surf zone conditions but mainly with steep beaches. Here it does not seem to overcome the performance of the other models, although it does a fairly good job, relatively improved, for rougher surf zone conditions and saturated ones.

\subsection{Numerical results}

Before focusing on the results for the sea-salt mass emission flux in the surf zone at Scripps' pier, we will first discuss the calculation of the emission flux for the test case reported by Neele et al. ${ }^{8}$ In this case, the inputs to the two wave models used in their analysis were a constant slope plane beach and an offshore wave field with significant wave height, $H_{s}$, equal to $0.6 \mathrm{~m}$ and wave period $T_{p}$ of $3.5 \mathrm{~s}$.

As mentioned in the introduction, Neele et al. ${ }^{8}$ claimed that one model, called ENDEC, which is based also on Battjes and Janssen's model provided more realistic values for the width of the surf zone and the cross-shore profile of wave energy dissipation in the surf zone in comparison with the predictions obtained with the model used by Chomka and Petelski ${ }^{6}$ that corresponds to our model $C$. We note that no details about the WEP models and definition of $L$ were presented and, further, that these results are somewhat in contradiction with past studies concerned by the analysis of the performance of WEP models for the surf zone. Moreover, it should be mentioned here that in the original paper of Chomka and Petelski ${ }^{6}$ many mistakes can be found in the wave formulas describing the WEP model used in their analysis.

Figures $3 \mathrm{a}$ and $3 \mathrm{~b}$ show the results obtained with our three models when applied to the test case studied by Neele et al. ${ }^{8}$. In contrast with the results reported by these authors, we find that the model $C$ performs as well as model $A$ and $B$, although it predicts lower values and different decay rates of $H_{r m s}$ well inside the surf zone due to the differences in the wave energy dissipation predictions. Figure 3a also shows that a definition of $L$ based on a threshold value of the wave energy dissipation as proposed by Chomka and Petelski would yield unrealistic large values. With our definition of $L$ we obtain $46 \mathrm{~m}$ with model $A, 47 \mathrm{~m}$ with model $B$ and $64 \mathrm{~m}$ with model $C$. To obtain the cross shore profile of the seasalt mass emission flux of Figure $3 b$, we have applied the CP formula (1) with the spatially averaged value of wave energy dissipation, $d E$, replaced by the local rate of wave energy dissipation $\varepsilon_{b}(\mathrm{x})$. The average quantity $d E$ is defined here as $d E=D_{b r} / L$, where $L$ is the length of the surf zone and $D_{b r}$ is total amount of wave energy dissipated that is calculated with 


$$
D_{b r}=\int_{0}^{x_{\max }} \varepsilon_{b}(x) d x
$$

Here $\mathrm{x}_{\max }=800 \mathrm{~m}$ is the distance from the shoreline to the furthest position offshore in the numerical domain and $\beta$ the slope of the beach was such as $\tan \beta=0.026$.

Figure $3 \mathrm{~b}$ shows that models $\mathrm{A}$ and $\mathrm{B}$ predict slightly higher maximal local values than those reported by Neele et al. ${ }^{8}$ using the ENDEC model. This can be explained because the ENDEC model is not similar to model $A$ or $B$ although it is also based on the Battjes and Janssen formulation. Indeed this model takes into account additional effects on the transformation of rms wave-height across the surf zone by including extra terms in the wave energy balance equation. These extra terms are related to vortical motions induced by the rollers formed beneath the sea surface after breaking. Without entering into details, these roller effects tend to reduce the amount of wave energy dissipation due to wave breaking and, thus, yield wider surf zone as well as smaller sea-salt mass emission flux. In Neele et al. ${ }^{8}$ the length of surf zone was estimated to be $230 \mathrm{~m}$ with the model ENDEC.

(a)
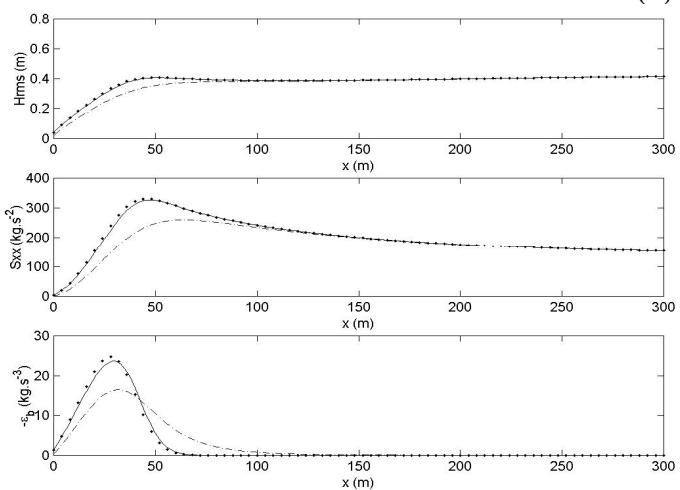

(b)
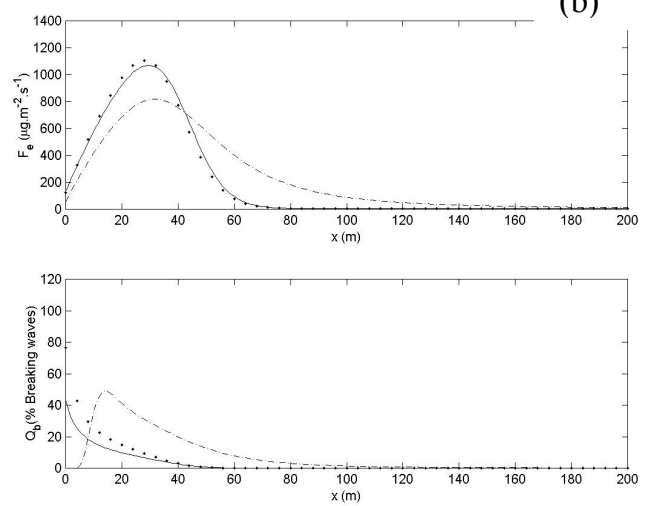

Figure 3: Comparison of wave model predictions: (a) profiles of $H_{r m s}$, set-up and dissipation; (b) profiles of sea-salt mass flux and local fraction of breaking waves in percentage. Dotted line: model $A$; Solid line: model $B$; Dashdotted line: model $C$.

Despite the differences in the local profiles and the width of the surf zone, it is remarkable that our predictions for the total average emission flux (TAF) per unit of length of shoreline (along shore) are in good agreement with the predictions of ENDEC used by Neele et al. ${ }^{8}$. The TAF is defined as $\mathrm{TAF}=\mathrm{Fe} \cdot L$ and is in units of $\mu \mathrm{g} \cdot \mathrm{m}^{-1} \cdot \mathrm{s}^{-1}$. In fact, we have found the TAF values of $3.8 \cdot 10^{4}$ with model $A$, $3.9 \cdot 10^{4}$ with model $B$ and $4.2 \cdot 10^{4}$ with model $C$. These values should be compared with the value of $3.7 \cdot 10^{4}$ reported by Neele et al. ${ }^{8}$. The values of the total amount of wave energy dissipated, $D_{b r}$, are also very similar to each other. Namely, we have found $D_{b r}=783 \mathrm{~kg} . \mathrm{m} . \mathrm{s}^{-3}$ with model $A, D_{b r}=782 \mathrm{~kg} . \mathrm{m} . \mathrm{s}^{-3}$ with model $B$ and $D_{b r}=784 \mathrm{~kg} . \mathrm{m} . \mathrm{s}^{-3}$ with model $C$, to be compared with the value of $421 \mathrm{~kg} . \mathrm{m} . \mathrm{s}^{-3}$ which was estimated from the data of Neele et al. ${ }^{8}$. Those results suggest that although the models differ in predicting the cross-shore profiles of the wave transformation, they yield similar predictions for the total wave energy dissipation and total sea-salt mass production due to the surf zone.

Using as inputs the wave data of the buoy CDIP073 mounted at the end of Scripps' pier and assuming a mean bottom slope equal to 0.03 with a plane beach with normally incident wave conditions, we have applied the CP method to estimate the total sea-salt mass flux induced by the surf zone over the period of the experiment. Figures $4 \mathrm{a}$ and $4 \mathrm{~b}$ show samples of the estimates obtained the three different models presented before. Figure 4a shows again that the three models yield very similar predictions for the 
width of the surf zone $L$ and the average emission flux $F_{e}$. As explained above and as it can be seen in figure $4 \mathrm{a}$, the differences in the predictions of $L$ accounts for most of the observed differences in the predictions of $F_{e}$. This is further illustrated by figure $4 \mathrm{~b}$, which shows that indeed the predictions for the global quantities $D_{b r}$ and TAF are almost identical independently of the details of the WEP formulation. Note that this conclusion relies on a definition of the breaker point based on the occurrence of a local maximum in the radiation stresses as the waves shoal into shallower waters.

(a)
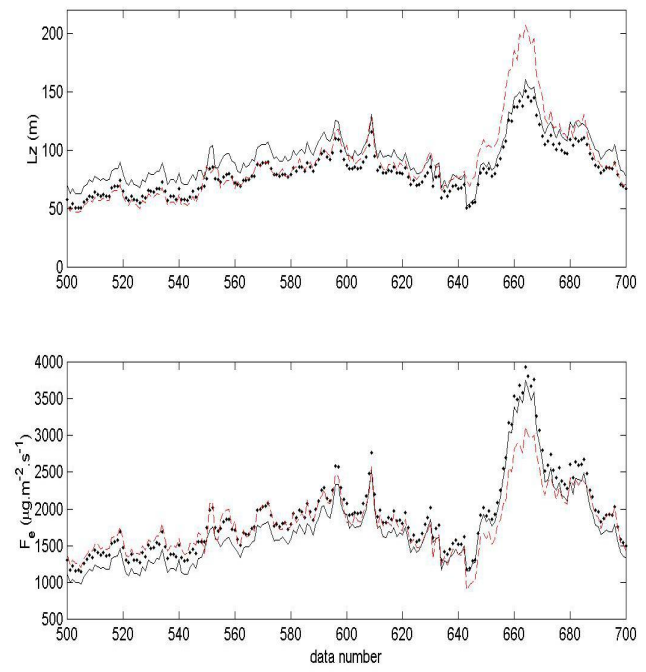

(b)
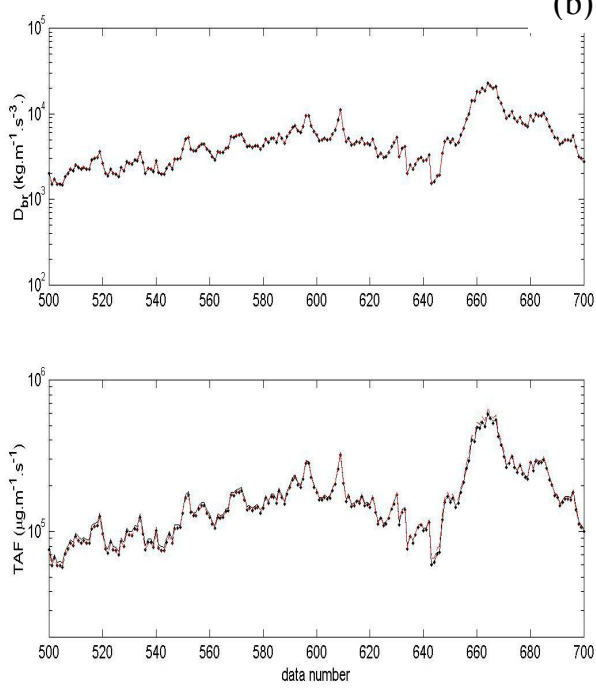

Figure 4: Predictions of three different wave models. Dotted line: model A; Dashed line: model B; Solid line: model C (a) for the width of the surf zone $L$ and the average sea-salt mass flux $F_{e}$ (b) for the total wave energy dissipated $D_{b r}$ and the total average sea-salt mass flux TAF.

The results presented here will be compared with the experimental estimations of the sea-salt emission flux $F_{e}$ using measurements of aerosol concentrations at Scripps's pier and will be reported elsewhere. The predicted $F_{e}$ values range from 600 to $3950 \mu \mathrm{g} \cdot \mathrm{m}^{-2} \cdot \mathrm{s}^{-1}$. These values can be compared with the values obtained by Chomka and Petelski ${ }^{6,7}$ using impactors measurements at two Baltic coastal sites in the range $3-384 \mu \mathrm{g} \cdot \mathrm{m}^{-2} \cdot \mathrm{s}^{-1}$, and the higher values reported by De Leeuw et al. ${ }^{3}$ using PMS measurements for the Californian coasts in the range $562-1034 \mu \mathrm{g} \cdot \mathrm{m}^{-2} . \mathrm{s}^{-1}$ for La Jolla and $297-858$ $\mu \mathrm{g} . \mathrm{m}^{-2} . \mathrm{s}^{-1}$ for Moss Landing. Few plausible explanations have been proposed to explain the differences between the observations at and at the Baltic coasts. Here, we speculate that these differences might not be so large, would the first proposed empirical coefficients of equation (1) have been overestimated by Petelski and Chomka ${ }^{6,7}$. This could have resulted from a misleading use of the wave model while attempting to correlate wave energy dissipation to observed sea-salt mass flux. This issue is under investigation and our results will be reported in a future publication.

\section{CONCLUSION}

The results of our work show that the aerosol emission model considered in this paper is not sensitive to the choice of the wave model that is used to calculate the wave energy dissipation due to wave breaking across the surf zone. With our definition of the width of the surf zone, it appears that although the local predictions of the wave transformation differ from one model to the other, the predictions for the total wave energy dissipated in the surf zone and the total aerosol flux per unit of length of shoreline (using the CP formula) are quasi independent of the details in the formulation of the wave model. 
The predictions of sea-salt mass flux obtained with the CP model will be tested again experimental values determined from measurements of the increase (compared to background) in size-distribution of SSA concentrations measured at the shore during onshore wind conditions. In view of the differences in the predicted values of $F_{e}$ reported here and its relative importance in relation to the total annual production of SSA along coastlines, we hope to clarify the performance of the model and eventually revisit the parameterization proposed by $\mathrm{CP}$ based on measurements along the Baltic coasts.

\section{REFERENCES}

1. Clarke, A.D. and Kapustin, V.N., The Shoreline Environment Aerosol Study (SEAS): A Context for Marine Aerosol Measurements Influenced by a Coastal Environment and Long-Range Transport. J. Atmos. Oceanic Technol., 20, 1351-1361, 2003.

2. Zielinski, T., Dependence of the surf zone aerosol on wind direction and wind speed at a coastal site on the Baltic Sea, Oceanologia 45(3), pp. 359-371, 2003.

3. De Leeuw, G., Neele, F.P., M. Hill, Smith, M.H., and E. Vignati, Production of sea spray aerosol in the surf zone, J. Geoph. Res., 105, D24, pp. 29,397-29,409, 2000.

4. Clarke, A.D., Owens, S.R, and Zhou, J., An ultrafine sea-salt flux from breaking waves: implications for cloud condensation nuclei in the remote marine atmosphere, J. Geophys. Res. 111, D06202, doi: 10.1029/2005JD006565, 2006.

5. Petelski, T. and Chomka, M., Marine aerosol fluxes in the coastal zone - BAEX experimental data, Oceanologia 38(4), pp.469-484, 1996.

6. Chomka, M. and Petelski, T., Modelling the sea aerosol emission in the coastal zone, Oceanologia 39(3), 211-225, 1997.

7. Petelski, T. and Chomka, M., Sea salt emission from the coastal zone, Oceanologia 42(4), pp. 399410, 2000.

8. Neele, F.P., Leeuw, de G., Neele, F.P., Jansen, M. and Stive, M., Quantitative assessment of surfproduced sea spray aerosol, in Propagation and imaging trough the Atmosphere II, edited by L.R. Bissonnette, Proc. SPIE Int. Soc. Opt. Eng., 3433, 53-62, 1998.

9. Battjes, J.A. and Janssen, J.P., Energy loss and setup due to breaking of random waves, Proc. $16^{\text {th }}$ Int. Conf. Coast Eng. ASCE, New York, pp. 569-588, 1978.

10. Thornton, E. B. and R. T Guza, Transformation of wave height distribution, J. Geophys. Res. 88, 5925-5938, 1983.

11. Battjes, J.A., Stive, M.J.F., Calibration and verification of a dissipation model for random breaking waves. J. Geophys. Res. 90, 9159- 9167, 1985.

12. Baldock, T.E., Holmes, P., Bunker, S., Van Weert, P., Crossshore hydrodynamics within an unsaturated surf zone. Coast. Eng. 34, 173-196, 1998.

13. Nairn, R.B., Prediction of cross-shore sediment transport and beach profile evolution. $\mathrm{PhD}$ thesis, Dept. of Civil Engineering, imperial College, London, 391 pp., 1990.

14. Thornton, E. B. and R. T Guza, Surf zone longshore currents and random waves: field data and models, J. Phys. Oceanogr., 16, 1165-1178, 1986.

15. Longuet-Higgins, M.S. and Stewart, R.W., A note on wave set-up, J. Mar. Res., 21, 4-10, 1963.

16. Lafon, C., Piazzola, J., Forget, P., Le Calve, O. and Despiau, S., Analysis of the variations of the whitecap fraction as measured in a coastal zone, Boundary-Layer Metorol., 111, 339-360, 2004.

17. Massouh, L. and Le Calve, O., Measurements of whitecap coverage during FETCH 98 experiment, J. Aerosol Sci., 30, Suppl. 1, 177-178, 1999. 